\title{
Information Visualization: The Need for a Data Base Approach
}

\author{
J Foley \\ Graphics, Visualization \& Usability Center \\ Georgia Institute of Technology, Atlanta GA 30332-0280 USA \\ 404853 0671, foley@gvu.gatech.edu
}

\begin{abstract}
Information visualizations data base technology. This is because the explosion in computer-stored information requires new ways to visualize information. We can no longer ask users to work with low-level graphics tools to create information presentations. Rather, we want users to be able to ask, at a high level, for visualizations which will help with a particular task, or will emphasize a certain trend. In order to automatically create such presentations, meta-data about the data will be needed. We give two examples of systems in which meta-data enables automatic generation of visual views: a dialogue-box generation tool for user interfaces and data entry, and a WWW visual navigation tool.
\end{abstract}

\section{INTRODUCTION}

My basic thesis is that the data base comunity and the information visualization community need to work together to realize the full potential of information visualization. I assert this thesis because based on a strong belief that in the future more and more information presentations will be automatically generated. Hence the basis for the thesis becomes clear. We can't do much to automatically visualize information which within the computer is not understood in some way or another: data base technology is the key way to represent such understandings. The schemas which define data properties and relationships are exactly what is needed to allow visualizatons to be created automatically.

Why are automatic visualizations desirable? The amount of computer-based information is growing rapidly, probably exponentially. With disk drives now costing around $\$ 500$ per gigabyte, more and more data is being maintained online. The rapid growth of the WWW is another indicator of how much digital information exists. Some portion of the online information is already managed by DBMSs; more will be. Indeed, the WWW itself is a very simple (too simple, we will argue) structured data base.

Given all the data being stored, it becomes less and less reasonable for end users to get involved in the details of creating charts and graphs. While Microsoft's Wizards simplify the process, I prefer to think further ahead to a time when the user will be able to make requests like "create for me a graphic which helps me understand the relationship between our inventory, our production schedules, our order backlog, and our profitability." This is where rich data descriptions become important. 
When browsing the WWW, we have essentially no use of graphics to show the user overview diagrams or roadmaps of the information space, no use of graphics to show where the user is in the information space or how to get to other locations, no use of graphics to distinguish between different types of nodes or links. Why is this? In part because the cost of creating such graphics manually, and then updating them whenever the content of the web changes, is an impossible task. Yet there is ample evidence that graphical navigational tools can be helpful. Again, rich data descriptions can pave the way for automatic generation and updating of these graphics.

In a somewhat different domain, objects as found in OODBMSs or as found in the new programming environment represented by IDL and CORBA are described by a meta language. For example, in the case of CORBA, the language is IDL. Each object is a package of data and procedures (methods) which operate on the data. As objects proliferate, the task of creating visual views of the objects again becomes daunting. But, I will argue, if the descriptive language is rich enough, then it will be possible automatically to create views of the objects which allow their data to be examined and their methods to be invoked.

To make these points more concrete, I will discuss two examples drawn from research projects conducted at the GVU Center which endeavor automatically to create visualizations.

\section{AUTOMATED GENERATION OF DIALOGUE BOXES}

The first example concerns automatic generation of user interface dialogue boxes which allow the user to create, edit, delete, and in general manipulate data objects. A semantic data model for an application is represented using Delft Univeristy's D2M2 [van der Mast 1993, Versendaal 1995] data base system.. The data and methods for each object are described in the D2M2 schema, using a dialogue-box fill in editor.

A rule-driven generation approach is used, driven by meta-information in the semantic data model. Design rules for dialogue boxes from Sun's Open Look style guide [Sun Microsystems and AT\&T, 1990] were encoded in a machine-processable form, and were then used to automatically select approriate widgets for dialogue boxes [deBaar, Foley and Mullet, 1992]. Figure 1 shows the overall system framework.

The key insight from this work is that standard data declarations (integer, float, character, enumerated are not enough to make good design decisions. Consider the enumerated data type and the way in which the end user might select members from an enumerated set. In OPEN LOOK, no less than seven different widgets (radio buttons and several styles of scrolling lists and check boxes) can be used for an enumerated type, depending on the number of elements in the set, whether the user must choose one element versus may choose one element versus may choose more than one element, and finally whether the elements of the set are bound at design time or are variable at run time. Hence the declaration of an enumerated set must not only delineate the members of a set, but this other information as well. This meant enriching the data declarations beyond those of D2M2, specifically for the purpose of choosing appropriate user interface constructs. (Note that more recent user interface toolkits and styleguides are equaly rich in providing many widget choices to the designer, using criteria similar to those of OPEN LOOK.) 


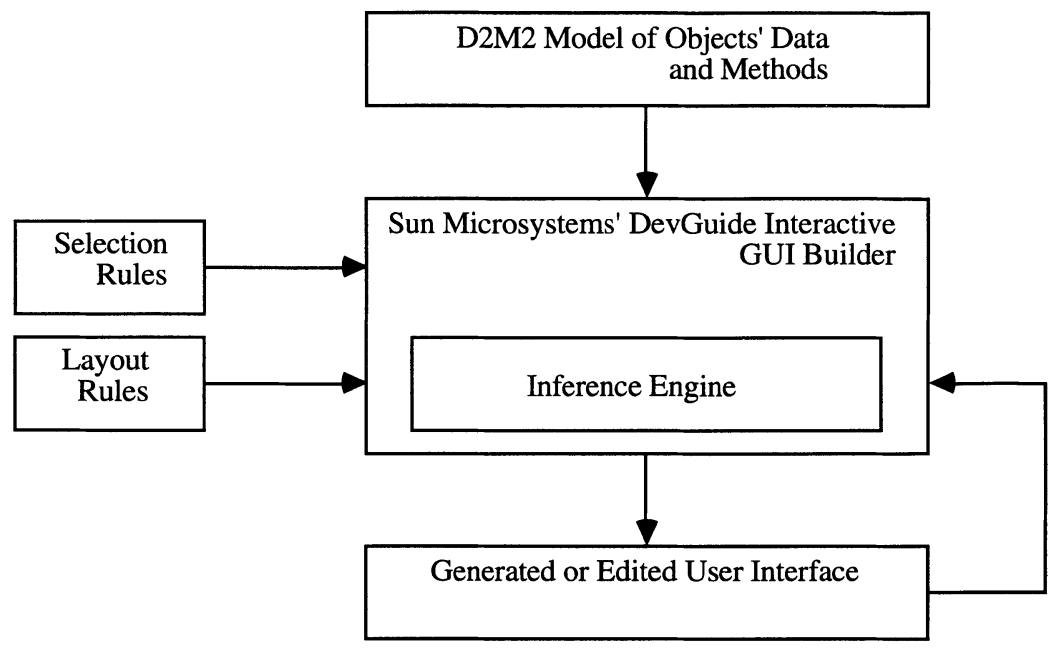

Figure 1 System for automatic layout of interfaces to objects. The data model for a specific object is selected and brought into D2M2. The selection and layout rules, encoded as humanreadable files, are processed by the inference engine and used to select appropriate widgets. The widgets are placed in the generated dialogue-box style interface using placement rules.

\section{AUTOMATIC GENERATION OF WORLD-WIDE WEB NAVIGATIONAL VIEWS}

In a second related endeavor, we developed layout (placement) rules to determine in what order to position widgets in the dialogue boxes [Gray, Foley and Mullet, 1994]. Clearly one can use the order of declaration in the data model, but that order may be random or may be more derived from the analysts' view of the data model than from end-user considerations. In our work, we were already using pre- and post-conditions to control the visibility of windows and the enabling of widgets (disable widgets being gray-toned in most user interface toolkits). Very often, these preand post-conditions cause actions on one widget to affect another widget. We reasoned that if widget $B$ is enabled by an action on widget $A$, then widget $B$ should be somewhere below widget $A$ in the dialoge box.. As well, the data model explicitly shows hierarchical groupings (such as a $3 D$ position type being an $x$-coordinate, a $y$-coordinate, and a $z$-coordinate, all of type coordinate.) We reasoned that the hierarchies should be conveyed to the user by visual grouping or indenting. Again, it is only by having a rich data model that such decisions can be made. 
The second example is visualizing the World-Wide Web, to facilitate navigation and exploration. We have developed a series of visualizations of the Web as part of NVB, the Navigational View Builder [Mukherjea and Foley CHI 1994] that use queries, abstraction, and visual bindings to reduce the visual clutter that occurs when many pages and links are shown. However, some of the NVB capabilities require that the HTML be supplemented with meta-data describing page and link categories (types) and subcategories. We have added this meta-data manually to selected sets of web pages for experimentation, but what is desperately needed is that HTML be extended to include meta-data explicitly. Since we started this project in 1993, the web community has perceived the meed for such meta-data, and has underway the Uniform Resource Characteristics project [Internet Engineering Task Force 1995] to enhance HTML to accomodate various types of augmentations, such as meta-data. Of course, another strategy would be to embed web pages in an OODBMS.

One visualization method we use is presenting a series of more and more detailed views, essentially working down an abstraction hierarchy such as might be represented in a class hierarchy. Ultimately, instances are shown. For instance, one hierarchy is [research center $\rightarrow$ research group $\rightarrow>$ research topic $\rightarrow$ research paper].

Another visualization method is binding the visual attributes of nodes and links in a graph-like diagram of a set of WWW pages to data values or meta-data concerning the nodes and links. For example, links of type "is advisor of" might be shown in green lines, while links of type "is author of" might be shown in red lines. Similarly, research topic nodes might be circles while research group nodes might be squares, etc.

Other types of visualizations are reported in several papers [Mukherjea, Foley and Hudson Hypermedia Technology 1994, Mukherjea, Foley and Hudson SGICHI 1995, Mukherjea and Foley WWW 1995]. The common thread is that they in general require descriptive data about data in order to be used, or in order to be used well. Again, DBMS technology is an appropriate technology to bring to bear.

\section{REFERENCES}

deBaar, D., J. Foley and K. Mullet, Coupling Application Design and User Interface Design, Proceedings CHI'92 - SIGCHI 1992 Computer Human Interaction Conference, ACM, New York, NY, 1992, pp. 259-266.

Foley, J. and J. Pitkow, eds. Report of the NSF Workshop on WWW Research Issues, in preparation, http://www.cc.gatech.edu:80/gvu/nsf-ws/nsf-ws.html.

Gray, M., J. Foley and K. Mullet, Grouping and Ordering User Interface Components, GVU Center Technical Report 94-03, Georgia Institute of Technology, Atlanta, GA 1994.

Internet Engineering Task Force 1995, Uniform Resource Identifiers, URL:http://www.gatech.edu/iiir/, 1995.

Mukherjea, S. and J. Foley, Navigational View Builder: A Tool for Building Navigational Views of Information Spaces, CHI '94 Conference Companion, p. 289-290.

Mukherjea, S., J. Foley and S. Hudson, Interactive Clustering for Navigating in Hypermedia Systems, Proceedings of the ACM European Conference on Hypermedia Technology, pp 136145, September 1994, Edinburgh, Scotland.

Mukherjea, S., J. Foley and S. Hudson, Visualizing Complex Hypermedia Networks through Multiple Hierarchical Views, Proceedings of ACM SIGCHI '95, May 1995, Denver, Colorado. 
Mukherjea, S. and J. Foley, Visualizing the World-Wide Web with the Navigational View Builder, Proceedings WWW Conference, Darmstadt, Germany, April 1995.

Sun Microsystems and AT\&T, Open Look GUI Application Style Guidelines, Addison-Wesley, Reading MA 1990.

van der Mast, C. and J. Versendaal, Separation of User Interface and Application with the Delft Direct Manipulation Manager, in: H.G. Stassen (Editor), Aanalysis, Design and Evaluation of Man-Machine Systems 1992, Pergamon Press 1993, ISBN 0-08-041900-3, p. 163-168.

Versendaal, J., W. Beekman, M. Kruit, and C. van der Mast, Architecture and Use of D2M2, the Delft Direct Manipulation Manager, in: C. Laffra, E.H. Blake, V. de Mey, X. Pintado, Object-

Oriented Programming for Graphics, Springer-Verlag, 1995, ISBN 0-387-58314-9, p. 136-151.

\section{ACKNOWLEDGEMENTS}

Dennis deBaar, Mark Gray, Sougata Mukherjea, and Kevin Mullet, as co-authors of the papers used as examples, contributed insights which have influcenced and enhanced my understandings of the issues discussed here. Thanks to Digital Equipment Corporation and Sun Microsystems for their financial support of these two projects.

\section{BIOGRAPHY}

Jim Foley earned the BS in Electrical Engineering from Lehigh University in 1964, the MS in EE from the University of Michigan in 1964, and the Ph.D. in Computer, Information, and Control Engineering from the University of Michigan in 1969. Prior to joining Georgia Tech in 1991, he served on the faculties of the University of North Carolina and The George Washington University, where he was most recently Chairman of the Department of Electrical Engineering and Computer Science. At Georgia Tech, he is the founding Director of the Graphics, Visualization \& Usability Center, which brings together over 30 faculty and 100 students from 10 different colleges, schools and departments for interdisciplinary research, teaching, and service. 